\section{Improving mammogram and breast sonography interpretation using computer-aided diagnosis}

A recent study has shown that the use of a computer-aided multimodality intelligent workstation improves interpretation of mammograms and breast sonograms, and the differentiation between benign and malignant lesions.

Five breast radiologists and five breast imaging fellows assessed images from a total of 97 biopsy-confirmed lesions, and gave confidence ratings (rating their confidence that the lesion was malignant on a continuous scale of $1-100 \%)$ and patient management decisions (follow-up or biopsy) without and with computer aid. Analysis showed that the average performance of the 10 observers was improved with use of the computer aid. There was a significant increase in area under the receiver operating characteristic $(\mathrm{ROC})$ curve $\left(A_{\mathrm{z}}\right)$ value $(0.87-0.92 ; P<0.001)$, partial $A_{\mathrm{z}}$ value $(0.47-0.68 ; P<0.001)$, and sensitivity $(0.88-0.93 ; P=0.005)$, although the difference in specificity with and without computer aid did not reach significance (0.66-0.69; $P=0.20)$. Overall, more observers changed their treatment decision correctly (i.e. from biopsy to follow-up for benign lesions, or follow-up to biopsy for malignant lesions) following computer-aided analysis than changed it incorrectly. Only one malignant lesion was incorrectly diagnosed as benign by the computer program, while four lesions were incorrectly diagnosed by at least one observer without computer aid. The authors conclude that further validation of this promising system in the clinical situation is warranted.

Original article Horsch K et al. (2006) Classification of breast lesions with multimodality computer-aided diagnosis: observer study results on an independent clinical data set. Radiology 240: 357-368

\section{Combined estrogen plus testosterone therapies increase the risk of invasive breast cancer}

Despite epidemiological studies suggesting a link between endogenous testosterone levels and increased risk of breast cancer in postmenopausal women, the use of hormone therapies containing androgens in such women is increasing.
Tamimi et al. conducted a prospective cohort study to examine the relationship between the use of testosterone-containing postmenopausal hormone $(\mathrm{PMH})$ formulations and invasive breast cancer. They identified 4,610 cases of invasive breast cancer over 24 years of followup $(1,359,323$ person-years). Overall, the risk of breast cancer was $77 \%$ greater among current users of estrogen plus testosterone therapies, and $58 \%$ greater among current users of estrogen plus progesterone therapies, than among those who had never used PMHs; in comparison, estrogen-only therapy was associated with only a $15 \%$ increased risk.

Risk estimates for $\mathrm{PMH}$ users were greatest in women with a natural menopause. In this group, the risk of breast cancer amongst current users of estrogen plus testosterone was almost 2.5 times that of women who had never used PMHs; current use of estrogen and testosterone was associated with a significantly increased risk of breast cancer compared with estrogen-only therapy $(P=0.007)$, and a marginally greater risk than estrogen and progesterone therapy $(P=0.11)$. Among these women, those using PMHs with testosterone had a $17.2 \%$ (95\% Cl 6.7-28.7\%) increased risk of breast cancer per year of use.

Estrogen and testosterone therapies are, therefore, associated with a significantly increased risk of invasive breast cancer. The authors suggest that the use of such therapies in postmenopausal women, especially in the long term, should be reconsidered.

Original article Tamimi RM et al. (2006) Combined estrogen and testosterone use and risk of breast cancer in postmenopausal women. Arch Intern Med 166: 1483-1489

\section{Chemotherapy and radiotherapy equally effective adjuvant therapies for endometrial cancer}

Surgery is the mainstay of treatment for women diagnosed with endometrial cancer; however, the best adjuvant therapy for preventing disease recurrence following surgery is still a matter of some debate.

In a randomized study, Maggi et al. allocated 345 patients diagnosed with high-risk endometrial cancer (FIGO stage IcG3, Ila-bG3 with deep myometrial invasion of $\geq 50 \%$, or stage III) to either chemotherapy (cyclophosphamide, doxorubicin and cisplatin every 4 weeks for 5 